\title{
Relación entre parámetros océano-atmosféricos y la producción del café verde en Colombia
}

\section{Relationship between ocean-atmospheric parameters and green coffee production in Colombia}

NANCY VILLEGAS ${ }^{1}$

JUAN CARLOS BARRIENTOS ${ }^{2}$

IGOR MÁLIKOV'

Frutos inmaduros de café

(Cundinamarca, Colombia).

Foto: J.C. Barrientos

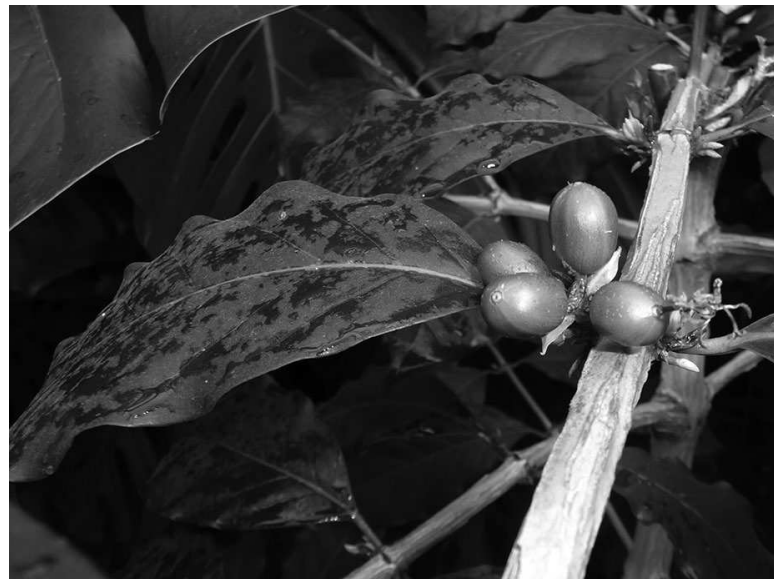

\section{RESUMEN}

Se determina la influencia de la migración de la zona de convergencia intertropical (ZCIT) y de El Niño oscilación del sur (ENOS) en la producción de café verde en Colombia durante el periodo de 1960 a 2007. Se dan a conocer las relaciones temporales entre la serie de producción de café verde (en grano y sin tostar) y las series que representan a los dos fenómenos de interacción océano-atmósfera. Para analizar la presencia de la oscilación de la ZCIT se utilizaron series de precipitación, la componente meridional del viento, la temperatura superficial del mar (TSM), la humedad relativa, la presión atmosférica y la temperatura del aire del puerto Buenaventura siendo el sector de Colombia donde se percibe su influencia. Los datos utilizados para el estudio de ENOS son las anomalías de TSM de las regiones Niño 1+2, Niño 3.4 y el índice oscilación del sur. Se concluye que la producción de café verde en Colombia está más influenciada por la migración de la ZCIT que por el ENOS.

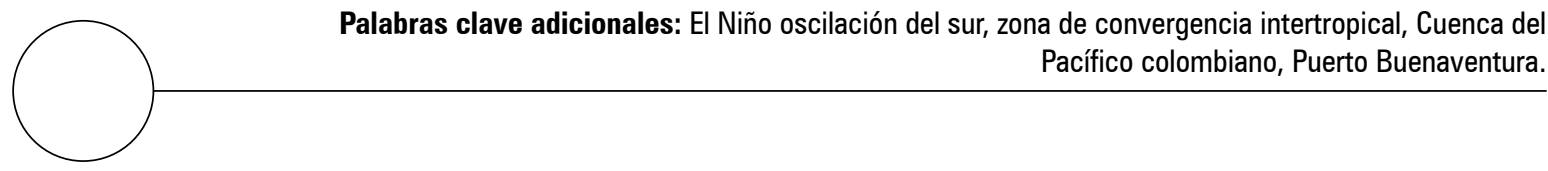

Facultad de Ciencias, Departamento de Geociencias, Grupo de Investigación CENIT, Universidad Nacional de Colombia, Bogotá (Colombia).

2 Facultad de Agronomía, Departamento de Agronomía, Universidad Nacional de Colombia, Bogotá (Colombia).

3 Autor para correspondencia: nlvillegasb@unal.edu.co 


\section{ABSTRACT}

The influence of the migration of the Intertropical Convergence Zone (ITCZ) and of the El Niño Southern Oscillation (ENSO) in the production of green coffee in Colombia (1960-2007) was determined. Temporal relationships between the production series of green coffee (unroasted beans) and the series representing the two phenomena of the ocean-atmosphere interaction were uncovered. To analyze the presence of the oscillation of the ITCZ, the following were used: precipitation series, the meridional component of wind, sea surface temperature (SST), relative humidity, atmospheric pressure and air temperature of the port of Buenaventura; the most influenced sector in Colombia. The data used for the study of the ENSO included the SST anomalies of the regions: Niño 1+2, Niño 3.4, and the Southern Oscillation Index. We conclude that green coffee production in Colombia is more influenced by the migration of the ITCZ than the ENSO.

Additional keywords: El Niño Southern Oscillation, Intertropical Convergence Zone, Colombian Pacific Basin, Port of Buenaventura.

Fecha de recepción: 13-01-2012

Aprobado para publicación: 29-05-2012

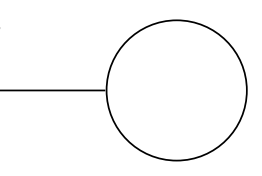

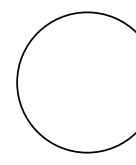

La climatología de Colombia está dominada por los desplazamientos sur-norte-sur de la zona de convergencia intertropical (ZCIT) (Von Prahl et al., 1990). La ZCIT es una región de baja presión orientada de oriente a occidente cerca al Ecuador, donde los vientos alisios del noreste se reúnen con los alisios del sureste (Devis et al., 2002). Esto hace que haya una abundante formación de nubes por convección, con vientos débiles y variables y alta pluviosidad. La ZCIT se desplaza latitudinalmente sobre el segmento del océano Pacífico oriental, correspondiente al territorio colombiano entre $1^{\circ}$ y $7^{\circ}$ de latitud norte en promedio, y en su desplazamiento, el sector por donde se percibe mayor tiempo su presencia es alrededor de los $3^{\circ}$ norte, donde se encuentra el puerto de Buenaventura (Villegas y Málikov, 2009). Sin embargo, durante eventos El Niñooscilación del sur esta posición extrema puede alcanzar los $5^{\circ}$ de latitud Sur aproximadamente (Zea et al., 2000). En Colombia se han realizado estudios acerca de la búsqueda de su influencia en aspectos físicos y dinámicos relacionados con la surgencia de aguas que permiten conocer zonas de producción de pesca, como en Díaz et al. (2008a, 2008b), donde se descubren zonas de surgencia que aumentan y disminuyen en intensidad durante el año en la cuenca del Pacífico colombiano (CPC) al paso de la ZCIT.

Estudios sobre las variables atmosféricas y oceanológicas y su relación con el fenómeno El Niño oscilación del sur (ENOS) se han realizado también para la región costera del Pacífico colombiano desde inicios de los años 90, lo cual se puede ver en Pabón y Montealegre (1992), donde se muestra la influencia del ENOS en el nivel del mar. Trabajos recientes han obtenido relaciones entre procesos naturales ocurridos en las zonas homogéneas de la CPC y entre los de las regiones Niño 1+2 y Niño 3.4, y el índice oscilación del sur (IOS) (Málikov y Villegas, 2005; Hernández et al., 2006a, 2006b) y la influencia de ENOS en la producción agraria. Uno de esos ejemplos es el que referencian Cadena et al. (2006) sobre la producción de palma africana en Tumaco. 
El café es uno de los productos más importantes de la agricultura colombiana. Para 2011 ocupó 921.060 ha (Federación Nacional de Cafeteros de Colombia, 2012), alrededor de una quinta parte del área cosechada del país y de una tercera porción de los cultivos permanentes. Su contribución al producto interno bruto (PIB) agropecuario fue de aproximadamente $11 \%$ en 2010 (Agronet, 2010), y aproximadamente al 19\% del empleo en la actividad agraria colombiana (MADR, 2011). De acuerdo con la Federación Nacional de Cafeteros de Colombia (2011) el valor de sus exportaciones está en promedio (20002010) en aproximadamente US $\$ 1.500$ millones anuales. Según esta misma fuente el área de cultivo no ha variado mucho en los últimos 10 años manteniéndose entre 850.000 y 900.000 ha. Sin embargo, la producción ha mostrado variaciones que van desde aproximadamente 12,6 millones de sacos de $60 \mathrm{~kg}$ en el 2007 a aproximadamente 7,8 millones de sacos en el 2009. Estas variaciones de la producción se deben a factores económicos, sociales y ambientales. Como ya se indicó anteriormente, en 2009 la producción cayó ostensiblemente. Las causas, según la Federación Nacional de Cafeteros de Colombia (2010), fueron el exceso de lluvias en un $40 \%$ que afectaron la floración y cuajado de frutos, la disminución del uso de fertilizantes por el alza de precios del petróleo y el incremento de la enfermedad de la roya. Galvis (2001) estimó la relación entre el PIB agrícola per cápita y algunas variables geográficas, y concluyó que en 1997 más del 80\% de la variabilidad de los niveles de productividad agrícola en los denominados "antiguos departamentos", se explica por la calidad de la tierra y por factores climáticos. Así se hace evidente la importancia de la influencia del clima en la producción de café.

Por la importancia de la producción de café verde en Colombia, la explicación encontrada en trabajos mencionados acerca la influencia de ZCIT y ENOS sobre aspectos físicos y dinámicos del Pacífico y sobre la producción de pesca en la CPC; y sobre la producción de palma africana en
Tumaco, se ha propuesto en el presente trabajo determinar la influencia que pueden tener estos dos fenómenos en la producción de café verde en Colombia.

\section{METODOLOGÍA}

Se han tomado como puntos geográficos de análisis a zonas del océano Pacífico donde los fenómenos de interacción océano atmósfera presentan mayor reacción en las variables de precipitación (Pr), la componente meridional del viento (V), la temperatura superficial del mar (TSM), la humedad relativa (HR), la presión atmosférica (PA), la temperatura del aire (TA), y la producción de café verde durante el periodo 1960-2007.

El área de estudio comprende un punto de observación dentro del litoral colombiano, el puerto de Buenaventura, de donde se tienen series de tiempo de los promedios mensuales de $\mathrm{Pr}, \mathrm{V}, \mathrm{Ta}$, $\mathrm{Hr}$, Pa y TSM, las cuales podrían esclarecer una influencia de la migración de la ZCIT. Además, con el fin de establecer las periodicidades de las series relacionadas con los eventos de El Niño oscilación del sur (ENOS) se tomaron series de anomalías de TSM (ATSM) de las regiones Niño 3.4 y Niño $1+2$ y los valores del índice de oscilación del sur (IOS) (figura 1).

La información utilizada corresponde a series mensuales del periodo 1960-2007 de las variables océano-atmosféricas del puerto Buenaventura tomadas del International Comprehensive Ocean-Atmosphere Data Set (ICOADS, 2010) de las regiones Niño 3.4 y Niño 1+2 obtenidas del archivo de la National Oceanographic and Atmospheric Administration (NOAA, 2010) del IOS (2010); y la información sobre producción del café verde en Colombia fue tomada de las estadísticas de la Federación Nacional de Cafeteros de Colombia (2011). Las series fueron estudiadas por medio del análisis espectral para la identificación de periodicidades características; el cálculo de la densidad espectral se realizó con el método 


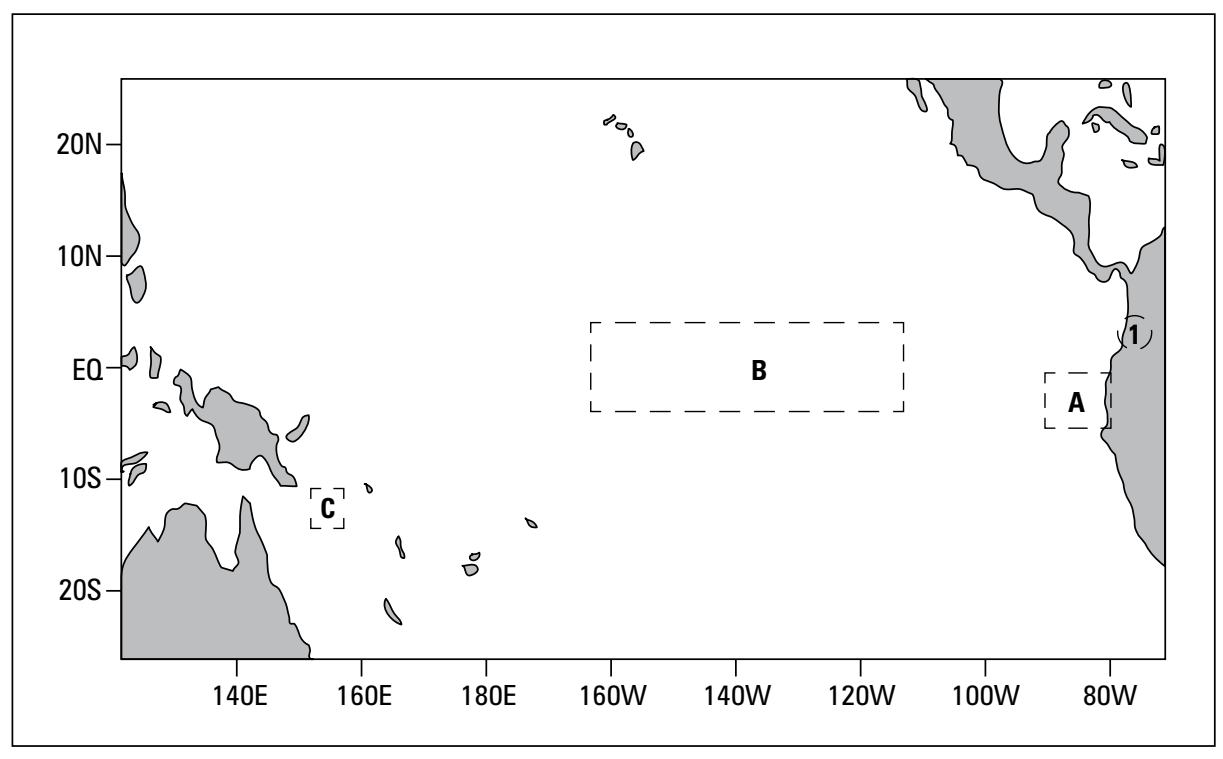

Figura 1. Área de estudio. 1. Puerto Buenaventura; A. Región El Niño 1+2; B. Región El Niño 3.4; C. IOS (modificado de Hernández et al., 2008).

de Furier; la extracción de componentes de cada serie se realizó con el filtro de Butterworth y la correlación se efectuó tanto entre series iniciales como entre sus componentes teniendo en cuenta rezagos en meses (Emery y Thomson, 2001).

\section{RESULTADOS Y DISCUSIÓN}

La producción del café verde en Colombia presenta desde 1960 una tendencia general de aumento en aproximadamente el doble hasta el año 1992; después se observa una disminución de la producción hasta el año 2003, desde entonces se registra otra vez un crecimiento de la producción (figura 2).

Con el fin de estudiar más detalladamente la serie de producción de café verde, se realizó el cálculo de la densidad espectral de la misma, observando que la estructura interna de la serie está formada por frecuencias con ciclos de 6 meses que da mayor aporte a la formación de las oscilaciones de la serie de la producción de café verde, después sigue el ciclo de 12 meses y finalmente se registra una tendencia de largo periodo de 12 años (figura 3). Los ciclos de 6 me- ses pueden explicarse por las dos cosechas que se realizan al año: abril-junio y septiembre-diciembre; y el de 1 año por la variación climática anual. Respecto del resultado de la tendencia de largo periodo se tiene menor certeza sobre los factores que lo determinan. Sin embargo, podría deberse, entre otros, 1) la introducción de nuevos cafetales, los que suelen producir frutos comercialmente rentables entre los 3 y 5 años después de su establecimiento en campo; 2) la renovación de cultivos maduros cada 6 a 7 años, y 3) los cambios atmosféricos relacionados con la ZCIT y de ENOS. Este último se analizará seguidamente.

Los ciclos más representativos de la figura 3 y de las gráficas de densidad espectral realizadas a las demás series analizadas, se muestran en la tabla 1. En ella se caracteriza la estructura interna de las series investigadas, donde los números de la izquierda corresponden a los valores más altos de densidad espectral encontrados en las series y hacia la derecha, los más bajos. Se ve que a nivel de 0,5 años la producción de café verde encuentra coincidencias de relación con precipitación, temperatura del aire, humedad relativa, presión atmosférica y temperatura superficial del mar. 
A nivel de 1 año con la precipitación, viento, temperatura del aire, humedad relativa, presión atmosférica y temperatura superficial del mar, y en el lapso de 12 años con la precipitación, el IOS, ATSM región Niño 1+2 y ATSM región Niño 3.4.

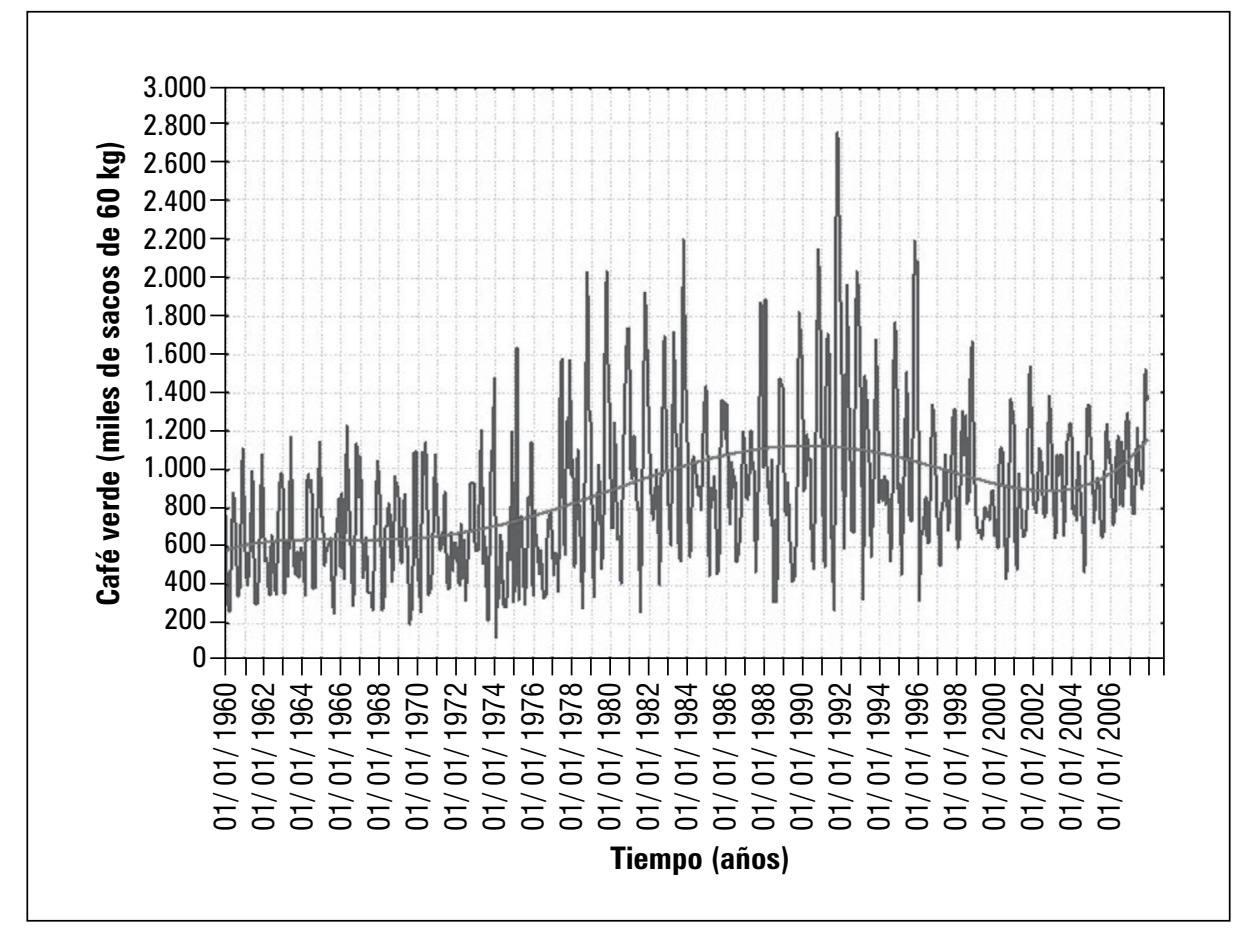

Figura 2. Serie inicial de la producción del café verde en Colombia para el periodo 19602007 (Federación Nacional de Cafeteros de Colombia, 2011).

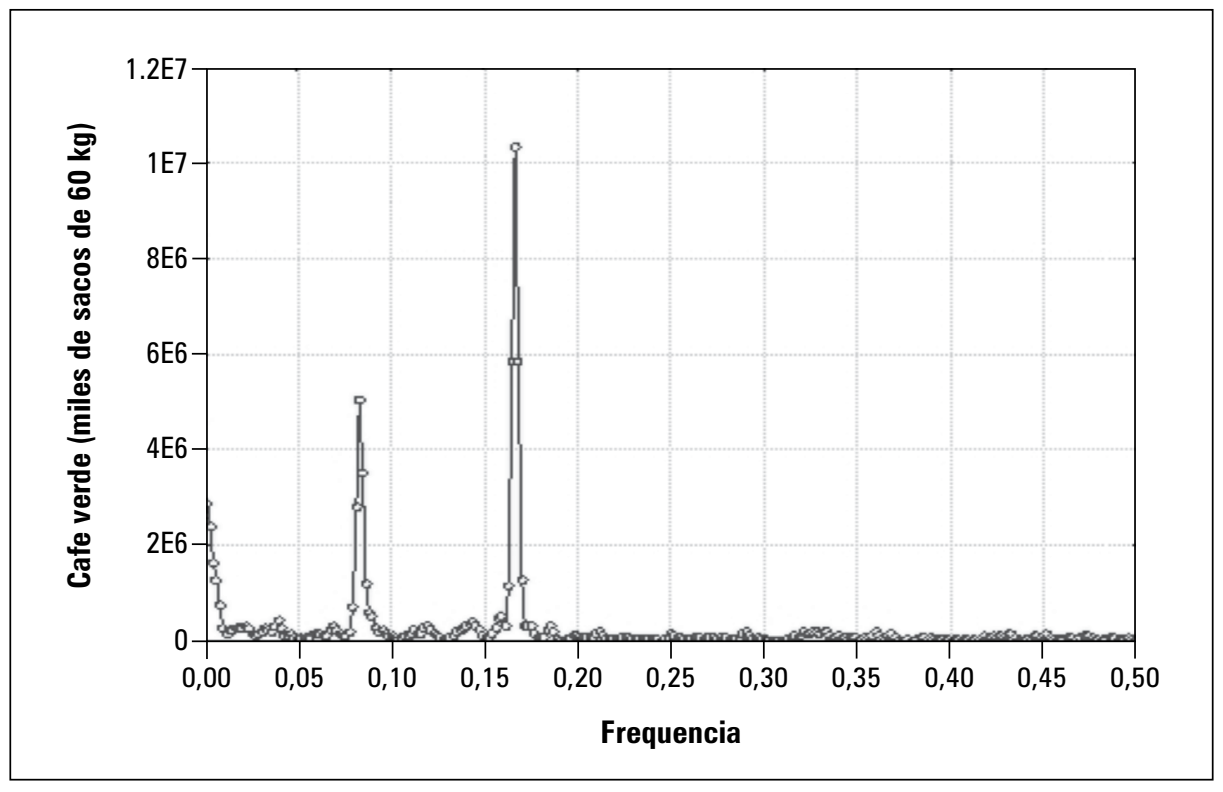

Figura 3. Densidad espectral de la serie del café verde. 
Tabla 1. Periodos encontrados con el análisis espectral de las series del periodo 1960-2007.

\begin{tabular}{|l|r|}
\hline \multicolumn{1}{|c|}{ Series analizadas } & \multicolumn{1}{|r|}{ Cuasi periodos (años) } \\
\hline Café verde & 0,$5 ; 1,0 ; 12$ \\
\hline Precipitación & 0,$5 ; 1,0 ; 12 ; 8 ; 3,7$ \\
\hline Componente V del viento & 1,$0 ; 16 ; 4,4$ \\
\hline Temperatura del aire & 1,$0 ; 0,5 ; 1,5 ; 12$ \\
\hline Humedad relativa & 0,$5 ; 1,0 ; 0,3 ; 5,3$ \\
\hline Presión atmosférica & 1,$0 ; 0,5 ; 16 ; 1,6$ \\
\hline Temperatura superficial del mar & 1,$0 ; 0,5 ; 2,4 ; 4,8 ; 24$ \\
\hline Índice de oscilación del sur & 4,$8 ; 3,7 ; 2,5 ; 12 ; 1,7$ \\
\hline ATSM Región Niño 1+2 & 3,$7 ; 4,8 ; 2,2 ; 12 ; 1,8 ; 0,9$ \\
\hline ATSM Región Niño 3.4 & 3,$7 ; 4,8 ; 2,5 ; 12 ; 1,8$ \\
\hline
\end{tabular}

Las relaciones temporales entre las series investigadas y sus componentes se presentan en la tabla 2 , donde en paréntesis se muestra, en meses, el rezago de la relación entre las series analizadas. Los coeficientes de correlación entre las series iniciales son bajos y no superan a 0,46. Las correlaciones de los cuasi periodos muestran mejores resultados. Las variables con las correlaciones más altas son: a los 0,5 años: precipitación; a 1 año: temperatura (inversa), presión atmosférica (inversa) y temperatura superficial del mar; y a los 12 años: precipitación, viento, temperatura del aire, IOS (inversa), Niño 1+2 y Niño 3.4.
En general, se observa que los coeficientes más altos están entre los componentes de mayor aporte en la serie investigada. El ciclo de 6 meses está relacionado con la migración de la ZCIT, la cual a su vez está asociada con la presencia de precipitación, presiones bajas, aumento de la HR, aumento de la TSM y disminución de TA. Como la ZCIT pasa dos veces al año sobre el territorio colombiano, sus efectos se ven reflejados en las series investigadas por ciclos de 6 y 12 meses. En el cuasi periodo de 12 meses los mejores coeficientes de correlación se observan entre el café y las series de viento, TA, PA y TSM. Las altas relaciones en las series de cuasi periodos de $6 \mathrm{y}$ 12 meses muestran que hay mayor influencia de la migración de la ZCIT que del ENOS. El cuasi periodo de 12 años está relacionado con la actividad solar (11 años en promedio), y esta con el aumento de nubosidad, y por ende con el aumento de lluvias en la zona ecuatorial. Las correlaciones más altas son: café versus series de precipitación, viento, Ta, IOS, Niño1+2 y Niño3.4.

\section{CONCLUSIONES}

Entre los dos fenómenos estudiados, el que presenta mayor influencia en la producción de café verde es la ZCIT.

Tabla 2. Coeficientes de correlación cruzada entre las series estudiadas para el periodo 1960-2007.

\begin{tabular}{|l|c|c|c|c|}
\hline \multirow{2}{*}{ Series correlacionadas } & \multirow{2}{*}{ Entre series iniciales } & \multicolumn{3}{|c|}{ Entre cuasi periodos (años) } \\
\cline { 3 - 5 } & & $\sim 0.5$ & $\sim 1$ & $\sim 12$ \\
\hline Precipitación vs. café & $0,46(-1)^{1}$ & $0,79(-1)$ & $0,19(1)$ & $0,72(0)$ \\
\hline Viento vs. café & & & $0,50(-4)$ & $0,51(-36)$ \\
\hline Temperatura del aire vs. café & $0,22(3)$ & $0,31(-2)$ & $-0,73(-2)$ & $0,68(36)$ \\
\hline Humedad vs. café & $-0,17(3)$ & $0,50(0)$ & $-0,43(3)$ & $-0,63(2)$ \\
\hline Presión atm. vs. café & $-0,13(3)$ & & $-0,79(0)$ & \\
\hline TSM vs. café & $0,18(-6)$ & & & $-0,74(0)$ \\
\hline IOS vs. café & $-0,27(-9)$ & & & $0,61(24)$ \\
\hline Niño 1+2 vs. café & $0,20(-12)$ & & & $0,62(0)$ \\
\hline Niño 3.4 vs. café & $0,22(-6)$ & & & \\
\hline
\end{tabular}

${ }^{1}$ Meses del rezago de la relación entre las series analizadas. 
La ZCIT está representada por presencia de precipitación, presiones bajas, aumento de la $\mathrm{HR}$, aumento de la temperatura del mar y disminución de la temperatura del aire.

La producción de café es más sensible a las variables meteorológicas: precipitación, $\mathrm{HR}$, temperatura del aire, TSM y presión atmosférica.

La precipitación tiene una alta correlación y está directamente relacionada con la producción de café verde.

La temperatura del aire, la temperatura superficial del mar y la presión atmosférica tienen una alta correlación y están inversamente relacionadas con la producción de café verde.

E1 ENOS presenta una mayor influencia en los ciclos de largos periodos como el de 12 años relacionados con IOS, la TSM de las regiones del pacífico Niño 1+2 y Niño 3.4, lo cual quiere decir que este ciclo propio del ENOS está superpuesto sobre el ciclo de la actividad solar.

Se recomienda estudiar a profundidad la relación de las variables precipitación-producción y presión atmosférica-producción con datos puntuales para cada región cafetera de Colombia.

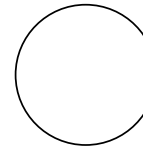

Agronet. 2010. Boletín de coyuntura económica: PIB 2(2). Ministerio de Agricultura y Desarrollo Rural. Bogotá 3 p. En: http://201.234.78.28:8080/ jspui/bitstream/123456789/1640/1/PIB Agropecuario_2010_I.pdf; consulta: septiembre de 2012.

Cadena, M., J. Ortiz, I. Málikov, J. Reyna, D. Pabón y A. Devis. 2006. Relationship between the 1997/98 El Nino and 1999/2001 La Nina events and oil palm tree production in Tumaco, Southwestern Colombia. Adv. Geosciences 6(1), 195 - 199.

Devis, A., I. García, I. Málikov y N. VILLEGAS. 2002. Compilación oceanográfica de la Cuenca Pacífica Colombiana. CCCP, Bogotá.

Díaz, D., I. Málikov y N. Villegas. 2008a. Características de las zonas de surgencia de la cuenca del Pacífico colombiano y su relación con la zona de convergencia intertropical. Boletín Científico $\mathrm{CIOH} 26$, $59-71$.

Díaz, D., I. Málikov y N. Villegas. 2008b. Descripción de los ciclos de ascenso de las aguas de la cuenca del pacífico colombiano durante el año y su relación con factores meteorológicos asociados con la zona de convergencia intertropical. Revista Institucional Universidad Tecnológica del Chocó 27(2), 200-206.

Emery, W. y R. Thomson. 2001. Data analysis methods in physical oceanography. $2^{\text {nd }}$ ed. Elsevier, Amsterdam, The Netherlands.

\section{REFERENCIAS BIBLIOGRÁFICAS}

Federacion Nacional de Cafeteros de Colombia. 2011. Información estadística cafetera. En:http:// wWw.federaciondecafeteros.org/particulares/es/ quienes_somos/119_estadisticas_historicas; consulta: abril de 2011.

Federacion Nacional de Cafeteros de Colombia. 2010. El comportamiento de la industria cafetera colombiana durante el 2009. Boletín Federecafé (Bogotá), 3-4, 11.

Federacion Nacional de Cafeteros de Colombia. 2012. Productores y cultivos de café en Colombia. En: http://www.federaciondecafeteros.org/particulares/es/quienes_somos/119_estadisticas_historicas/; consulta: septiembre de 2012 .

Galvis, L.A. 2001. خQué determina la productividad agrícola departamental en Colombia? Documentos de Trabajo sobre Economía Regional No. 19. Banco de la República, Sucursal Cartagena, Colombia.

Hernández, D., I. Málikov y N. Villegas. 2008. Respuestas de la temperatura superficial del mar y del aire en la cuenca del Pacífico colombiano producidas por El Niño oscilación sur. Ingenieria de Recursos Naturales y del Ambiente (Universidad del Valle) $1(7), 56-64$.

Hernández, D., I. Málikov y N. Villegas. 2006a. Relaciones espacio-temporales entre la temperatura superficial del mar de la Cuenca del Pacífico Colombiano y el ciclo El Niño oscilación del sur. En: Memorias 
VII Congreso Colombiano de Meteorología (en CD). 5-7 Junio de 2006. Bogotá.

Hernández, D., I. Málikov y N. Villegas. 2006b. Respuestas de la temperatura superficial del mar y la temperatura del aire de la Cuenca del Pacífico colombiano ante El Niño oscilación del sur. En: IV Encuentro RUPSUR 2006 (en CD). 8-10 Noviembre de 2006. Cali, Colombia.

Índice oscilación del sur (IOS). 2010. En: http://www. bom.gov.au/climate/glossary/soi.shtml; consulta: diciembre de 2010 .

International Comprehensive Ocean-Atmosphere Data Set (ICOADS). 2010. En: http://icoads.noaa.gov; consulta: diciembre de 2010.

Málikov, I. y N. Villegas. 2005. Construcción de series de tiempo de temperatura superficial del mar de las zonas homogéneas del océano Pacífico colombiano. Boletín Científico CCCP 12, 79-93.

Ministerio de Agricultura y Desarrollo Rural (MADR). 2011. Perspectivas agropecuarias-primer semestre del 2011. Bogotá.
Ministerio de Agricultura y Desarrollo Rural (MADR) - Observatorio Agrocadenas Colombia. 2005. La cadena del café en Colombia. Una mirada global de su estructura y dinámica 1991-2005. Documento de trabajo 59. Bogotá.

National Oceanographic and Atmospheric Administration (NOAA). 2010. En: http://www.cpc.ncep noaa.gov/data/indices; consulta: diciembre de 2010.

Pabón, J. y J. Montealegre. 1992. Manifestación El Niñooscilación del sur (ENOS) en la costa Pacífica colombiana. Boletín ERFEN 31, 3-11.

Villegas, N. e I. Málikov. 2009. Atlas de características termohalinas y dinámicas de las aguas de la cuenca del Pacífico Colombiano: con base en simulación matemática. Uniblios, Universidad Nacional de Colombia, Bogotá.

Von Prahl, H., J. Cantera y R. Contreras. 1990. Pacífico Colombiano. Fondo para la Protección del Medio Ambiente, FEN, Bogotá.

Zea, J., J. Eslava y G. León. 2000. Circulación general del trópico y la zona de confluencia intertropical en Colombia. Meteorol. Colomb. 1, 31-38. 